Verona, Italy; ${ }^{9}$ Division of Rheumatology, Fondazione Policlinico Universitario A. Gemelli IRCCS, Università Cattolica del Sacro Cuore, Rome, Italy, Rome, Italy; ${ }^{10}$ Rheumatology Unit, Department of Internal Medicine and Medical Speciality, Sapienza University of Rome, Rome, Italy; ${ }^{11}$ Rheumatology Unit, University of Messina, Messina, Italy

Background: Systemic lupus erythematosus (SLE) patients are at high risk for CV events, and EULAR recommends assessing the 10-year CV-risk using the Systematic Coronary Risk Evaluation (SCORE) [1]. The QRISK3, another score to assess CV-risk in UK population, considers different factors among which also SLE. The Progetto Cuore score (PCS) is validated to estimate CV risk in Italian people and largely replicates the SCORE project [2].

Objectives: This cross-sectional study aimed to estimate CV-risk using SCORE, QRISK3 and, for the first time, PCS in a multicentric cohort of Italian SLE patients. Methods: During 2019 we evaluated 173 SLE patients (87.7\% female; age $40 \pm 16$ years; disease duration $138 \pm 105$ months), fulfilling the 1997 ACR classification criteria. Clinical and laboratory data were registered, and individual CV-risk was calculated using suitable algorithms for the SCORE, QRISK3 and PCS. Statistical analysis was performed using Graphpad Instat 8.0 (San Diego, CA-USA).

Results: In $13(7 \%)$ SLE patients a previous CV event was recorded. Hypertension was present in $60(37.5 \%)$ and diabetes in $27(16.9 \%)$ patients. Mean total cholesterol was $184 \pm 39 \mathrm{mg} / \mathrm{dL}$, HDLc $58 \pm 18 \mathrm{mg} / \mathrm{dL}$, LDLc $124 \pm 37 \mathrm{mg} / \mathrm{dL}$, triglycerides $105 \pm 63 \mathrm{mg} / \mathrm{dL}$; dyslipidaemia was reported in $58(36.2 \%)$ patients and $29(18.1 \%)$ were on statin. Mean BMI was $24.9 \pm 5.3 \mathrm{Kg} / \mathrm{sm}, 60(37.5 \%)$ and $23(14.3 \%)$ patients were overweight and obese, while 25 (15.6\%) patients were smokers. $87(54.3 \%)$ SLE patients had a SLEDAl $<4,91 \%$ of patients were taken $\mathrm{HCQ}$ and $65 \%$ were on prednisone (mean dose $5.4 \pm 5.9 \mathrm{mg} /$ day), but only $7.5 \%$ took $>7.5 \mathrm{mg} /$ day. The CV-risk of SLE patients according to SCORE, QRISK3 and PCS was $1.1 \pm 2.1 \%, 10.5 \pm 12.3 \%$ and $3.7 \pm 5.4 \%$, respectively. Stratifying patients at low, moderate or high $\mathrm{CV}$ risk according to the PCS and SCORE a double proportion of patients was at moderate ( $8 \%$ vs $3.9 \%$ ) or high $(1.9 \%$ vs $0.9 \%) \mathrm{CV}$ risk ( $p=0.03$ ). Finally, CV-risk according to QRISK3 was higher than $20 \%$ (high risk) in $32 / 160(20 \%)$ patients.

Conclusion: This multicentre study demonstrated that the mean estimated CV-risk in SLE patients is globally low using the SCORE, QRISK3 and PCS. The PCS seems to better intercept those patients at moderate/high risk, at least in Italian SLE patients, while QRISK3 predicts the highest CV risk. The lack of disease-specific CV-risk factors (such as autoantibodies profile or organ involvement) probably account for the underestimation of $\mathrm{CV}$ risk using the SCORE and PCS.

References:

[1] ARD 2019;78(6):736-745.

[2] ARD 2019;0:1-2. doi:10.1136/annrheumdis-2019-215715

Disclosure of Interests: Fabio Cacciapaglia Speakers bureau: BMS; Roche; Pfizer; Abbvie, Andreina Manfredi: None declared, Gianluca Erre: None declared, Elena Bartoloni Bocci: None declared, Garifallia Sakellariou Speakers bureau: Abbvie, Novartis, MSD, Ombretta Viapiana: None declared, Sergio Colella: None declared, Anna Abbruzzese: None declared, Marco Fornaro: None declared, Giacomo Cafaro: None declared, Maria Antonietta Fenu: None declared, Bianca Lucia Palermo: None declared, Martina Dessì: None declared, Adalgisa Palermo: None declared, Alessandro Giollo: None declared, Elisa Gremese Consultant of: AbbVie, Bristol-Myers Squibb, Celgene, Eli Lilly, Janssen, Merck Sharp \& Dohme, Novartis, Sanofi, UCB, Roche, Pfizer, Speakers bureau: AbbVie, Bristol-Myers Squibb, Celgene, Eli Lilly, Janssen, Merck Sharp \& Dohme, Novartis, Sanofi, UCB, Roche, Pfizer, Francesca Romana Spinelli Grant/research support from: Pfizer, Speakers bureau: Lilly, BMS, Celgene, Fabiola Atzeni: None declared, Matteo Piga: None declared

DOI: 10.1136/annrheumdis-2020-eular.6187

\section{THU0258 \\ SJOGREN'S SYNDROME WITH AND WITHOUT AND AUTOIMMUNE THYROIDITIS: IS THERE ANY DIFFERENCE?}

A. I. Celia ${ }^{1}$, S. Colafrancesco ${ }^{1}$, A. Gattamelata ${ }^{1}$, R. Izzo ${ }^{1}$, F. Giardina ${ }^{1}$, L. Mastromanno', R. Priori'. ' 'Sapienza University of Rome, Dipartimento di Scienze Cliniche, Internistiche, Anestesiologiche e Cardiovascolari, Rome, Italy

Background: Sjögren's syndrome (SS) is a systemic autoimmune disease mainly affecting exocrine glands and characterized by a progressive lymphocytic infiltration of salivary and lacrimal glands with consequent loss of function and development of sicca symptoms. Autoimmune thyroiditis (AT) is the most frequent autoimmune disease associated with SS and detectable in about $10-30 \%$ of cases ${ }^{1}$. Interestingly, patients with concomitant SS and AT seem to display a more attenuated phenotype compared to patients with solely SS. It is also noteworthy that up to $30 \%$ of patients with AT experience sicca symptoms without a clear diagnosis $\mathrm{SS}^{2}$. At the light of these evidences, it is unclear whether SS and AT represent two distinct nosological entities or different expressions of the same pathology.

Objectives: Aim of this study is to evaluate the prevalence of AT in a large monocentric cohort of patients with SS and to define its clinical and laboratory characteristics compared to isolated SS.

Methods: Consecutive patients with SS (AECG criteria) referring to our "Sjögren Clinic" (Sapienza University of Rome) were enrolled and divided in two groups: SS with AT (group 1) and SS without AT (group 2). Group 1 was further divided in two subgroups depending on the presence (1a) or absence (1b) of anti Ro/SSA antibodies. The following clinical and laboratory data were retrospectively collected for all patients: concomitant celiac disease, arthralgia, lung involvement, purpura, lymphoma, presence of ANA, anti-Ro/SSA, anti-La/ SSB, rheumatoid factor, cryoglobulins, leukopenia and hypergammaglobulinaemia. These characteristics were compared between the following groups: group 1 , group 2, group $1 \mathrm{a}$ and $1 \mathrm{~b}$. For statistic Chi Square and Fisher's test analysis were performed.

Results: Six-hundred and three SS patients were enrolled (group $1 \mathrm{n=135}$; group $2 \mathrm{n}=381$; group 1a $\mathrm{n}=96$; group $1 \mathrm{~b} \mathrm{n}=39$ ). The prevalence of AT was 135/603 (22.3\%). When comparing SS patients with or without AT (group 1 vs group 2) the frequency of rheumatoid factor was significantly higher in group 2 compared to group $1(p=0.006)$. No case of lymphoma was recorded in group 1 while 14 cases of lymphoma were ascertained in group $2(p=0,08)$. Conversely, celiac disease was higher in group 1 compared to group $2(p=0.01)$. No other differences between these groups were identified. Stratifying SS patients with AT according to the presence (group 1a) or not (group 1b) of anti Ro/SSA antibodies, ANA rheumatoid factor and hypergammaglobulinemia were significantly more positive in group 1 a compared to group $1 \mathrm{~b}(\mathrm{p}=0.0002, \mathrm{p}=0.002, \mathrm{p}=0.02$, respectively); no clinical differences were identified.

Conclusion: In this study, we confirm the presence of a less aggressive disease in patients with SS and AT compared to solely SS. The higher prevalence of rheumatoid factor and lymphoma occurrence in SS without AT, strictly suggest a more severe phenotype in this subset. Although is known that in SS patients with anti Ro/SSA+ antibodies and RF there is a more aggressive disease, in SS with AT the presence or absence of such autoantibodies do not seems to associate with any difference in clinical severity. Follow up studies are presently being carried out in order to provide conformation of a less sever phenotype and a better disease outcome in patients with associated SS and AT.

\section{References:}

[1] Jara, L.J., Navarro, C., Brito-Zerón, M.P. et al. Thyroid disease in Sjögren's syndrome. Clin Rheumatol 26, 1601-1606 (2007)

[2] Vera D. Milic, Goran Radunovic, Ivan Boricic, Sanja Ognjanovic, Radmila Petrovic, Marija Radak-Perovic, Nada Vujasinovic-Stupar, Nemanja Damjanov, High prevalence of autoimmune thyroid disease in subjects with sicca symptoms without Sjögren's syndrome, Rheumatology, Volume 52, Issue 4 April 2013, Pages 754-755

Disclosure of Interests: None declared

DOI: 10.1136/annrheumdis-2020-eular.6059

\section{THU0259 \\ LYMPHOMAS IN ANTICENTROMERE ANTIBODY POSITIVE PRIMARY SJOGREN'S SYNDROME}

B. Chaltcev ${ }^{1}$, V. Vasiliev ${ }^{2}$, S. Palshina ${ }^{1}$, J. Khvan ${ }^{1}$, E. Sokol ${ }^{1}$, A. Torgashina ${ }^{1}$

V.A. Nasonova Research Institute of Rheumatology, Moscow, Russian

Federation; ${ }^{2}$ MEDSI, Moscow, Russian Federation

Background: Patients with primary Sjogren's syndrome (pSS) are at high risk of lymphoma. Signs of lymphomas in ACA+pSS were not widely reported, the descriptions were single [1, 2]. According to pSS treatment recommendations [3, $4,5,6]$, glandular forms require only symptomatic treatment, in the absence of systemic manifestations systemic therapy is not required.

Objectives: to evaluate the incidence of lymphomas in ACA+pSS; to compare clinical and laboratory manifestations in 2 groups of ACA+pSS: with and without lymphomas.

Methods: we examined 119 ACA+pSS patients. We evaluated both glandular and systemic manifestations. We diagnosed lymphomas based on study of biopsy specimens of affected organs.

Results: MALT-lymphomas diagnosed in 19 (16\%) ACA+pSS patients. Persistent enlargement of parotid salivary glands, decreased C4-complement, decreased CD19+cells in peripheral blood, cryoglobulinemic vasculitis, lymphoid infiltration of minor salivary glands (MSG) more than 200 cells in focus, severe xerostomia and xerophthalmia more often detected in patients with ACA+pSS+lymphomas. RF and antiRo were found in only $25 \%$ of patients with lymphomas and its 
frequency did not differ between groups. Only $20 \%$ of patients with lymphoma had monoclonal immunoglobulins secretion and its frequency also did not differ between groups. Anemia, leukopenia, thrombocytopenia, increased ESR, hypergammaglobulinemia, increased levels of immunoglobulins were found in the study groups with the same frequency. There were no differences in the frequency of detection of recurrent parotitis, lymphadenopathy, Raynaud phenomenon, arthritis/arthralgia, pleuritis/pericarditis, neuropathy, nephritis, hypergammaglobulinemic purpura.

Conclusion: in the present study in patients with lymphomas, the course of pSS was characterized by minimal systemic manifestations and low immunological activity, but severe glandular manifestations with the development of late stage damages of salivary and lacrimal glands, severe lymphoid infiltration of MSG, which led to the frequent occurrence of MALT-lymphomas. Thus, in patients with pSS, regardless of the type of detected antibodies (antiRo/ $\mathrm{La}, \mathrm{ACA}, \mathrm{RF}$ or others), regardless of the presence or absence of systemic manifestations, damage of salivary and lacrimal gland progresses, which in some cases leads to the development of lymphomas, therefore, therapy that can prevent this complication should be initiated immediately after diagnosis of pSS is confirmed. The signs of lymphoproliferation detected in the present study should be evaluated in all ACA+pSS patients for early diagnosis of lymphoma.

References:

[1] Baldini C, Mosca M, Della Rossa A, Pepe P, Notarstefano C, Ferro F, Luciano N, Talarico R, Tani C, Tavoni AG, Bombardieri S. Overlap of ACA-positive systemic sclerosis and Sjögren's syndrome: a distinct clinical entity with mild organ involvement but at high risk of lymphoma

[2] Dhiraj Gulati \& Irving Kushner \& Elizabeth File \& Marina Magrey. Primary Sjogren's syndrome with anticentromere antibodies-a clinically distinct subset. ClinRheumatol (2010) 29:789-791 DOI 10.1007/s10067-009-1359-9.

[3] F. Vivino et al. Sjogren's syndrome: An update on disease pathogenesis, clinical manifestations and treatment. Clinical Immunology 203 (2019) 81-121. https://doi.org/10.1016/j.clim.2019.04.009

[4] Xavier Mariette et al. Primary Sjögren's Syndrome. N Engl J Med 2018; 378:931-9. DOI: 10.1056/NEJMcp1702514

[5] Xiaoyun Chen et al. Advances in the diagnosis and treatment of Sjogren's syndrome. Clinical Rheumatology (2018) 37:1743-1749. https://doi.org/10.1007/ s10067-018-4153-8

[6] Ana-Luisa Stefanski et al. The Diagnosis and Treatment of Sjögren's Syndrome. Deutsches Ärzteblatt International | Dtsch Arztebl Int 2017; 114: 354-61

Disclosure of Interests: None declared

DOI: 10.1136/annrheumdis-2020-eular.3572

\section{THU0260 $\quad$ GLUCOCORTICOID DOSE IS AN INDEPENDENT PREDICTOR OF MORTALITY IN PATIENTS WITH SYSTEMIC LUPUS ERYTHEMATOSUS HOSPITALIZED FOR INFECTION}

T. Y. J. Heng ${ }^{1}$, N. Chew ${ }^{2}$, K. A. Choo ${ }^{1}$, A. Lateef ${ }^{2}$, M. Lahiri ${ }^{2}{ }^{1}$ National University of Singapore, Yong Loo Lin School of Medicine, Singapore, Singapore;

${ }^{2}$ National University Hospital, Medicine, Singapore, Singapore

Background: Infection is an important cause of mortality and morbidity in patients with systemic lupus erythematosus (SLE) and a common cause for hospitalization. Glucocorticoids (GC) may contribute to increased mortality.

Objectives: We performed a 10-year retrospective study of SLE patients hospitalized for infection, and the clinical predictors of mortality, especially GC dose, in these patients.

Methods: Diagnosis codes for SLE were obtained from the electronic medical records for hospitalized patients from 2005 to 2015. Chart review was performed to ascertain the indication for hospitalization. The first hospitalization for infection (if any) was used as the index admission. Demographic and clinical characteristics, infection site and immunosuppressive drugs over the past month were abstracted. Multivariable logistic regression was used to determine predictors of all-cause mortality at 1 year.

Results: Diagnosis codes were obtained for 768 unique SLE patients with 3660 hospitalization episodes over 10 years, of which 689 had a physician diagnosis of SLE on chart review. Of these, $250(36 \%)$ had an index admission for infection. 243/250 (97.2\%) fulfilled the ACR 1997 criteria for SLE and were studied further (Figure 1). Median (IQR) age was $45.1(37.3,56)$ years, $86 \%$ were female, $72 \%$ were Chinese, median (IQR) disease duration was $9(4,17)$ years. $53(21.8 \%)$ patients had chronic kidney disease (CKD), 34 (14\%) had diabetes mellitus (DM) and $12(4.9 \%)$ had cancer. $231(95.1 \%)$ patients were on immunosuppressive drugs and $210(86.4 \%)$ were on GC. The median (IQR) GC dose was $8(5,15)$ $\mathrm{mg}$ oral prednisolone equivalent per day. The median (IQR) Systemic Lupus Erythematosus Disease Activity Index (SLEDAI) score was 4 (1, 10). The most common organism was Escherichia coli, followed by Staphylococcus aureus and
Salmonella enteritidis. The respiratory tract was the most common site of infection. $27.9 \%$ of patients who had blood cultures performed had bacteremia. There were 11 (4.5\%) ICU admissions, 6 (2.5\%) patients died in hospital and 1-year all-cause mortality was $13(5.3 \%)$. SLEDAI had only a weak positive correlation with $\mathrm{GC}$ dose $\left[\mathrm{R}^{2}=0.039 /\right.$ Pearson's correlation $\left.\left.=0.197, \mathrm{p}=0.004\right)\right]$ (Figure 2). Increased age (odds ratio (OR) $1.07,95 \% \mathrm{Cl} 1.02-1.12, \mathrm{p}=0.005$ ), average daily GC dose (OR 1.05, 95\% Cl 1.02-1.09, $\mathrm{p}=0.002$ ), bone infections (OR 42.24 $95 \% \mathrm{Cl} 2.76-646.8, \mathrm{p}=0.007$ ) and CKD (OR 4.78, 95\% Cl 1.06-21.54, $\mathrm{p}=0.04$ ) were independent predictors of 1-year mortality, after adjusting for gender, SLE DAI, DM, and cancer (Table 1).

Table 1. Predictors of mortality

\begin{tabular}{|c|c|c|c|c|}
\hline Factors & $\begin{array}{c}\text { Univariable } \\
\text { Odds Ratio } \\
(95 \% \mathrm{Cl})\end{array}$ & P-value & $\begin{array}{c}\text { Multivariable } \\
\text { Odds Ratio } \\
(95 \% \mathrm{Cl})\end{array}$ & P-value \\
\hline Age at admission (years) & $1.04(1.005,1.07)$ & 0.02 & $1.07(1.02,1.12)$ & 0.005 \\
\hline $\begin{array}{l}\text { Average dose of oral predni- } \\
\text { solone }(\mathrm{mg} / \text { day })\end{array}$ & $1.04(1.01,1.06)$ & 0.006 & $1.05(1.02,1.09)$ & 0.002 \\
\hline Gender (female vs male) & $3.52(1.22,10.12)$ & 0.02 & $0.37(0.07,2.10)$ & 0.26 \\
\hline Disease duration $>10$ years & $1.27(0.49,3.33)$ & 0.62 & & \\
\hline Positive blood culture & $0.54(0.17,1.75)$ & 0.30 & & \\
\hline Site of infection & 1 (reference) & 0.17 & & 0.06 \\
\hline \multicolumn{5}{|l|}{ Gastrointestinal } \\
\hline Respiratory & $1.09(0.28,4.29)$ & 0.90 & $0.71(0.09,5.57)$ & 0.74 \\
\hline Renal & $0.27(0.03,2.48)$ & 0.25 & $0.44(0.03,5.94)$ & 0.54 \\
\hline Skin, Gynecological, Other & $0.94(0.25,3.51)$ & 0.93 & $1.04(0.16,6.74)$ & 0.96 \\
\hline Bone & $11.75(1.29,107.1)$ & 0.03 & $42.24(2.76,646.8)$ & 0.007 \\
\hline Primary Bacteremia & $0.00(0.00,0.00)$ & 0.99 & $0.00(0.00,0.00)$ & 0.99 \\
\hline SLEDAI score $\geq 4$ & $1.23(0.47,3.21)$ & 0.66 & $0.65(0.15,2.74)$ & 0.56 \\
\hline Diabetes Mellitus & $0.46(0.14,1.50)$ & 0.20 & $1.61(0.31,8.3)$ & 0.57 \\
\hline Chronic Kidney Disease & $0.30(0.11,0.78)$ & 0.01 & $4.78(1.06,21.54)$ & 0.04 \\
\hline Cancer & $0.18(0.04,0.77)$ & 0.02 & $3.52(0.25,48.82)$ & 0.35 \\
\hline
\end{tabular}

Conclusion: Higher dose of oral GC was an independent predictor of mortality, even after adjusting for disease activity. It is important to prescribe the minimum effective dose of GC in SLE patients with infection, especially in older patients with CKD and bone infection.

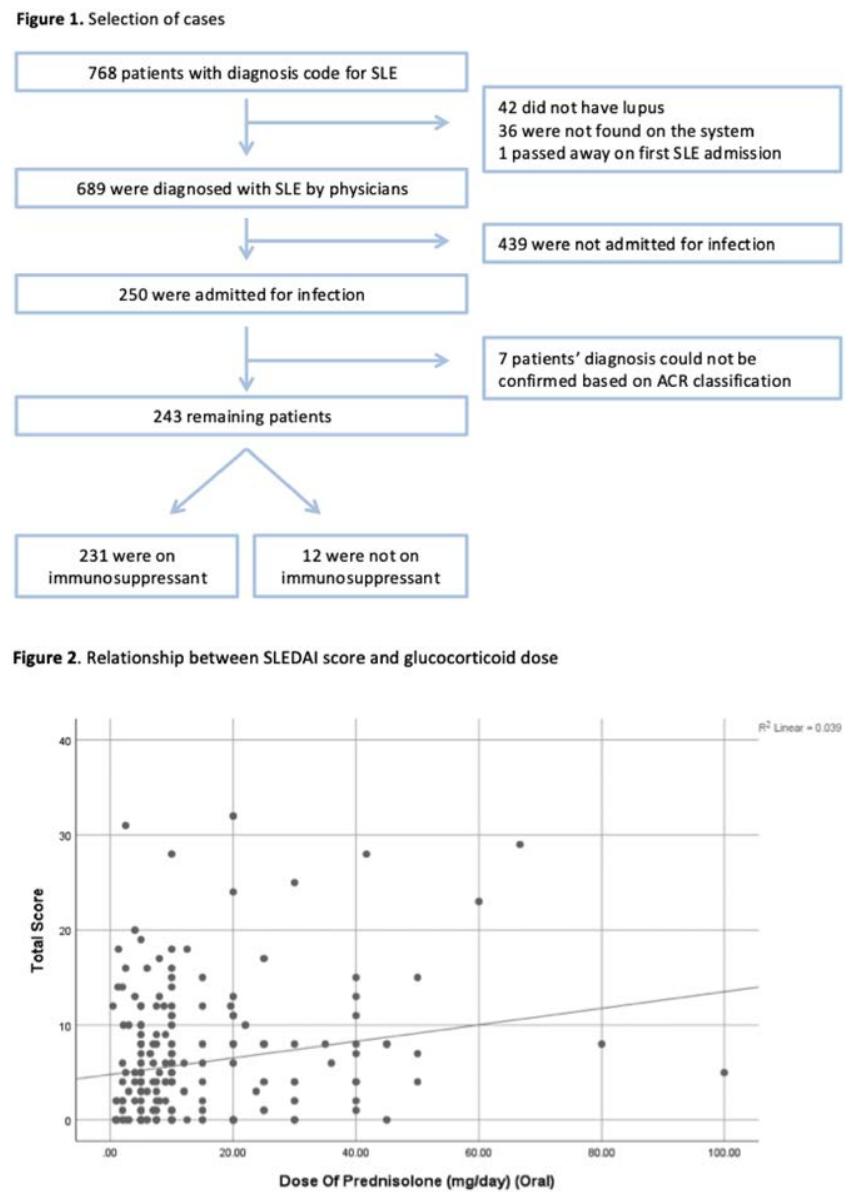

